\title{
REVERSE DYNAMISATION: A MODERN PERSPECTIVE ON STEPHAN PERREN'S STRAIN THEORY
}

\author{
V. Glatt ${ }^{1,4, *}$, C.H. Evans ${ }^{2}$ and K. Tetsworth ${ }^{3,4}$ \\ ${ }^{1}$ Department of Orthopaedics, University of Texas Health Science Centre, San Antonio, TX, USA \\ ${ }^{2}$ Rehabilitation Medicine Research Centre, Mayo Clinic, Rochester, MN, USA \\ ${ }^{3}$ Department of Orthopaedic Surgery, The Royal Brisbane and Women's Hospital, Brisbane, Australia \\ ${ }^{4}$ Orthopaedic Research Centre of Australia (ORCA), Brisbane, Australia
}

\begin{abstract}
The present review acknowledges the tremendous impact of Stephan Perren's strain theory, considered with respect to the earlier contributions of Roux and Pauwels. Then, it provides further insight by examining how the concept of reverse dynamisation extended Perren's theory within a modern context. A key factor of this more contemporary theory is that it introduces variable mechanical conditions at different time points during bone healing, opening the possibility of manipulating biology through mechanics to achieve the desired clinical outcome. The discussion focusses on the current state of the art and the most recent advances made towards optimising and accelerating bone regeneration, by actively controlling the mechanical environment as healing progresses. Reverse dynamisation utilises a very specific mechanical manipulation regimen, with conditions initially flexible to encourage and expedite early callus formation. Once callus has formed, the mechanical conditions are intentionally modified to create a rigid environment under which the soft callus is quickly converted to hard callus, bridging the fracture site and leading to a more rapid union. The relevant literature, principally animal studies, was surveyed to provide ample evidence in support of the effectiveness of reverse dynamisation. By providing a modern perspective on Stephan Perren's strain theory, reverse dynamisation perhaps holds the key to tipping the balance in favour of a more rapid and reliable union when treating acute fractures, osteotomies, non-unions and other circumstances where it is necessary to regenerate bone.
\end{abstract}

Keywords: Reverse dynamisation, dynamisation, interfragmentary strain theory, bone healing, fracture healing, mechanical environment, fixation stability, animal models.

Address for correspondence: Vaida Glatt, PhD, Assistant Professor, Department of Orthopedic Surgery, University of Texas Health Science Centre San Antonio, 7703 Floyd Curl Drive, MC 7774, San Antonio, TX 78229-3900, USA.

Telephone number: +1 2104508094 Email: glatt@uthscsa.edu

Copyright policy: This article is distributed in accordance with Creative Commons Attribution Licence (http://creativecommons.org/licenses/by-sa/4.0/).

\section{Introduction}

Across the full breadth of medicine and surgery there is a crucially important underlying relationship between biology and biomechanics, and nowhere is this more apparent than when observing fracture healing. Extensive clinical experience and numerous experimental studies throughout the past century have consistently supported one governing principle of fracture healing, that the biology can be suitably influenced to accelerate the progression towards union by providing the appropriate mechanical conditions (Augat et al., 2003; Augat et al., 2005; Carter et al., 1998; Cheal et al., 1991; Claes et al., 1998; Goodship et al., 1998; Kenwright and Gardner, 1998; Perren and Rahn, 1980). Whether treated by casting, traction, internal fixation, intramedullary nailing or external fixation, the healing response is predominately influenced by the stability of the fracture site. While the biological response undoubtedly reflects activities determined by local conditions, this response is largely driven by the mechanics of the system, thereby optimising the rate and probability of fracture healing. Therefore, the success of the healing process ultimately relies upon an intricate synergy between the biology and the mechanical environment (Augat et al., 2005; Carter et al., 1998; Hagiwara et al., 2015; Ignatius et al., 2005; Pauwels, 1960; Utvag et al., 2001; Utvag and Reikeras, 1998; Utvag et al., 1999).

The mechanical environment itself is determined by a combination of the stiffness of the fracture fixation device used, the magnitude of the loads applied, the properties of the tissue in the fracture site, as well as the types of loads allowed by the fixation device such as axial, shear or bending moments (Aro and Chao, 1993; Aro et al., 1990; Augat et al., 1998; Bishop et al., 
2006; Bottlang et al., 2010; Carter et al., 1988; Cheal et al., 1991; Claes et al., 1997; Claes and Heigele, 1999; Egger et al., 1993; Goodship and Kenwright, 1985; Goodship et al., 2009). Accordingly, whenever fixation is too flexible, the magnitude of interfragmentary motion (IFM) around the fracture site cannot be controlled and it will be subjected to high magnitude hybrid loading conditions including combinations of axial, shear and bending moments (Augat et al., 2003; Augat et al., 1996; Carter and Wong, 1988; Epari et al., 2006). Alternatively, if the fracture fixation is too rigid, it will prevent any micromotion within the fracture gap, resulting in bone resorption (Akeson et al., 1976; Chao et al., 1989; Matsushita and Kurokawa, 1998; Probst et al., 1999; Uhthoff and Dubuc, 1971; Utvag and Reikeras, 1998). In both circumstances, the activation of the biological processes will be suboptimal and, consequently, bone healing might fail.

The present review discusses the current state of the art and the advances made towards optimising and accelerating bone regeneration by controlling the mechanical environment as healing progresses. This includes an overview of Stephan Perren's strain theory and introduces a refined modernisation of his theory that uses a mechanical manipulation regimen called reverse dynamisation. A key factor of this contemporary theory is that it introduces variable mechanical conditions at different time points during bone healing, which opens the possibility of manipulating the biology to achieve a desired outcome. To fully comprehend how the mechanical environment can be effectively manipulated, it is important to first understand the essential biology differentiating normal from abnormal fracture healing.

\section{Biology of fracture healing}

The biology of fracture repair is a genuine wonder of nature and rather than healing by the production of a surrogate scar material, it is instead typically the result of true regeneration of adult tissue. Consequently, bone is one of the few organs in the body that has the capacity to heal without leaving a residual scar. Molecular events governing fracture healing reflect the activity of a relatively complex network of continuously changing signals in response to the initial tissue damage and necrosis, growth factor production, macrophage and stem cell activation, cell recruitment and migration, cell proliferation and differentiation, as well as tissue formation. Fracture healing typically involves three distinct phases, beginning with haematoma formation and inflammation (early phase), followed shortly after by an extended reparative phase and finally invoking a prolonged remodelling phase.

This process demands the coordinated activity of different cell populations in harmony with one another, each of which proliferates, differentiates and finally synthesises the requisite extracellular matrix components. During the inflammatory phase, the coagulation pathway plays an important role, and with the traumatic rupture of blood vessels a haematoma quickly forms between the bone ends, and the periosteum often lifts from the adjacent cortex. Many important signalling molecules are released within this haematoma, including inflammatory cytokines such as interleukin-1 (IL-1) and interleukin-6 (IL-6), and growth factors such as platelet-derived growth factor (PDGF), insulinlike growth factors (IGFs), and members of the transforming growth factor- $\beta$ (TGF- $\beta$ ) superfamily, including BMPs. Then, progenitor cells enter the fracture site to initiate repair, while the growth factors interact closely to regulate the initiation of fracture healing and any associated cellular response. These cells are derived from various locations, including the inner cambial layer of the periosteum, endosteum, bone marrow, surrounding musculature and vascular endothelium. As the progenitor cells differentiate into chondrocytes and osteoblasts, they initiate bone formation via either endochondral or intramembranous ossification, respectively.

With intramembranous ossification, or primary fracture healing, bone forms directly from osteoprogenitor cells to create hard callus, without an intervening cartilaginous phase. In contrast, endochondral ossification, or secondary fracture healing, first involves formation of a cartilaginous intermediate through the differentiation of progenitor cells into chondrocytes, that then becomes calcified and is eventually replaced by bone. This type of fracture healing provides an early, bridging, soft callus that is most often attributed to the adjacent periosteum. Mineralisation of this cartilaginous intermediate involves a mechanism similar to the growth plate associated with the growth of long bones and, therefore, requires reestablishment of the vascular and nutrient supply. The classical descriptions of this process report a sequence where chondrocytes hypertrophy and undergo apoptosis, the extracellular matrix calcifies and blood vessels penetrate the matrix. However, newer evidence has suggested that hypertrophic chondrocytes can transdifferentiate directly into osteoblasts (De La Vega et al., 2018; Hu et al., 2017; Yang et al., 2014). Either way, the provisional calcified cartilage is gradually resorbed and replaced by immature woven bone formed by osteoblasts. In the final healing phase, osteonal remodelling of the newly formed bone tissue, and of the fracture ends themselves, restores the original shape and the mature lamellar structure of the bone. This final phase of bone remodelling can take anywhere from months to years to complete and is profoundly influenced by mechanical loading conditions.

\section{Stephan Perren's interfragmentary strain theory}

Bone healing is a complex event requiring the coordination of a network of biological processes, 
where the local mechanical environment plays a crucial role. While the biological processes of bone healing have been studied extensively, less is known about the mechanical factors that play critical roles in determining healing outcomes. Much of the current appreciation of the regulative effect of mechanical forces on cell differentiation comes from Friedrich Pauwels (Pauwels, 1960). He refined concepts from the original ideas of early bone biologists such as Roux, who proposed that cells compete for functional stimulus within the tissue (Roux, 1881a; Roux, 1881b). Anticipating specific details that had not yet been discovered, Pauwels theory essentially proposed that whenever mesenchymal stem cells are subjected to high shear the functional stimulus would favour fibroblast differentiation, whereas endochondral bone formation would occur in low strain/high compression environments.

Perren subsequently proposed his theory that when the fractured bone is loaded, the fracture fragments displace with respect to each other and he introduced the term "interfragmentary strain" (IFS) to quantify the relative motion produced within the fracture gap (Perren and Cordey, 1980). The basis of his theory is that only the tissues that can withstand this IFS without rupturing can continue to exist in the fracture gap. IFS is defined as the magnitude of interfragmentary motion (axial) divided by the fracture gap size. The magnitude of the IFS determines the subsequent differentiation of fracture gap tissue, where the tissue cannot exist in an environment exceeding the strain tolerance of the extracellular matrix of these tissues (Fig. 1). Granulation tissue within a fracture can tolerate a strain of up to $100 \%$, and once granulation tissue is formed early callus will act to reduce the IFS. Then, this reduction in motion of the fracture fragments creates an environment more favourable for chondrogenesis. Therefore, if a strain higher than $10 \%$ (unstable fixation) persists, it is more likely to result in a disorganised tissue, typically fibrocartilaginous or cartilaginous in character, producing a hypertrophic or atrophic non-union that is unlikely to lead to bony union (Fig. 2a). When the strain is between 2 and $10 \%$ (semirigid/flexible fixation), it will be broadly conducive to woven bone formation through endochondral ossification, or secondary fracture healing. A strain lower than $2 \%$ (rigid fixation) will occur if the main fracture fragments are directly opposed and compressed against one another (Fig. 2b). These conditions are tolerated by lamellar bone and are regarded as characteristic of a rigid environment, leading to intramembranous ossification, or primary bone healing. Similarly, Claes and colleagues (Claes and Heigele, 1999; Claes et al., 1998) hypothesised that direct bone healing occurs with strains lower than approximately $5 \%$ and hydrostatic pressures lower than $\sim 0.15 \mathrm{MPa}$. Conversely, endochondral ossification is associated with compressive pressures higher than $\sim 0.15 \mathrm{MPa}$ and strains lower than $\sim 15 \%$. Considering relative motion across the fracture site, the IFS theory (IFST) frames issues regarding the stability of fixation with respect to the strain across the gap under a given load. In so doing, it defines convenient, if somewhat arbitrary, limits with respect to interfragmentary motion (IFM) and these limits broadly guide surgeons in terms of implant choice, implant placement and fracture reduction.

Since its introduction, Stephan Perren's work related to IFST has had a profound influence on experimental studies and orthopaedic trauma care for the past several decades, providing a conceptual link between the mechanics of fracture stabilisation and the biological response within and adjacent to a fracture gap (Perren, 1979; Perren, 2002; Perren, 2008; Perren et al., 2015; Perren and Rahn, 1980). Furthermore, this philosophy has been instrumental in the design of orthopaedic trauma implants for almost 50 years and has influenced several generations of orthopaedic surgeons. However, the shortcoming of the IFST is that it is simplistic and deterministic by its nature and fundamentally defines an "all or nothing" response. The role of mechanotransduction is implicit, but the theory itself sheds no light on the processes that may be responsible. More importantly, it does not consider the possibility of a
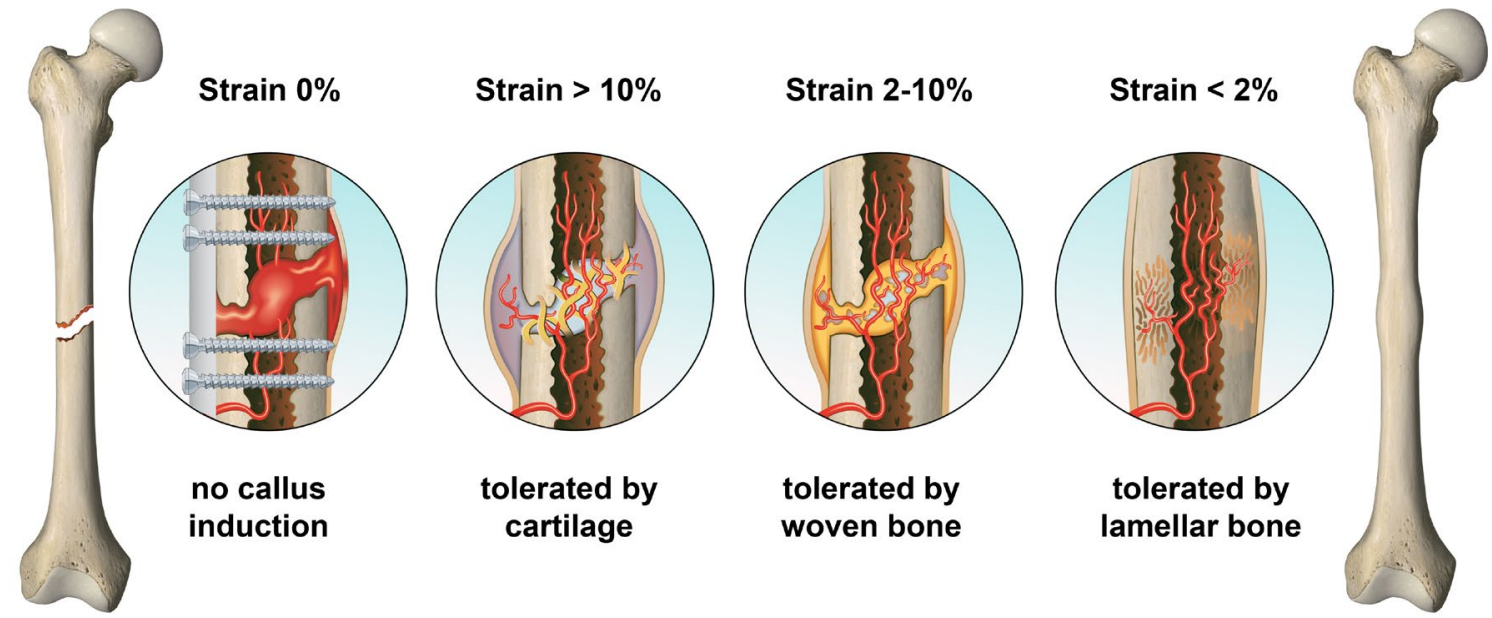

Fig. 1. Illustration demonstrating the magnitude of the interfragmentary strain tolerated by specific tissue types in the fracture gap. 
temporal evolution as the system responds and does not allow for any change in the mechanical conditions that inevitably result from the ensuing biological response. These limitations do not diminish the validity of the message and it remains conceptually attractive while appearing to coincide with the easily verifiable biological response to specific imposed mechanical conditions. Its simplicity is perhaps most responsible for its widespread adoption as the currently accepted model and for being recognised as one of the most fundamental principles of fracture fixation.

Nevertheless, moving away from the parameters of the IFST in its purest form, it is possible to explore the implications of the interplay between biology, mechanics and how activity influence fracture healing. The biology of fracture healing can be elegantly summarised conceptually by the two musical terms of harmony and melody. Harmony reflects the behaviour of two or more biological processes, acting simultaneously at any given point in time. For example, when two or more notes interact, the single sound that is perceived is quite specific and its pitch is inevitably very highly constrained, with very narrow bounds between what is regarded as either pleasing or unpleasant. While harmony is representative of the biological response at any given point in time, melody is instead representative of the biological response over time. However, a melody can gradually progress either as a coordinated response to defined conditions or can instead become amelodic and progress without direction, without form and, therefore, remain unlikely to proceed to a satisfying outcome.

Following this line of thinking, biology is, in a sense, fundamentally local in character and is, therefore, also exquisitely sensitive to very specific properties on the smallest scales. Consequently, processes such as stem cell recruitment and activation, inflammation, angiogenesis, fibrogenesis, chondrogenesis and osteogenesis may, or may not, be actively engaged at any given time, depending on local conditions, and most often are coordinated by their response to the mechanical environment. All of the many biological responses contribute together to the activity recognised as fracture healing, both at the local and at the global levels. As a synchronised behaviour, fracture healing will often be a result of the coordinated response of these biological processes proceeding to solid bone union. Conversely, a chaotic, discordant biological response would more likely fail to heal spontaneously, resulting in fibrous tissue and non-union.

It would be very difficult to predict from one condition alone whether or not it is possible to achieve optimal fracture healing. Within this conceptual framework there are, of course, many possibilities, but according to the IFST, the initial conditions reflecting the strain in the fracture gap either are or are not conducive to bone healing. By introducing variable conditions at different time points during bone healing, it is possible to control the mechanical environment to purposefully influence the biology. This conceptual freedom essentially opens the possibility of actively manipulating a fracture to achieve the desired outcome, bony union. Knowing this, is it possible to manipulate the fracture site to rapidly, predictably and consistently accelerate and optimise fracture healing? Yes, if Stephan Perren's strain theory is modernised by means of the novel mechanical regimen called reverse dynamisation (Fig. 3).

\section{Reverse dynamisation}

Using the basic IFST, experimental and clinical studies have attempted to optimise and accelerate the bone healing process using dynamisation. In this process the stiffness of the fixation is converted from a rigid to a more flexible configuration to encourage healing progression to solid bone union. This philosophy is based on the belief that by dynamising a fracture in this manner, the bone is trained to gradually accept more load while still being protected by fracture fixation. However, results using conventional dynamisation in both animal models (Claes et al., 2011; Claes et al., 2009; Egger et al., 1993; Goodship et al., 1998; Larsson et al., 2001; Utvag et al., 2001; Utvag and Reikeras, 1998; Utvag et al., 1999) and clinical studies (Basumallick and Bandopadhyay, 2002; Domb et al., 2002; Huang et al., 2012; Litrenta et al., 2015; Papakostidis et al., 2011; Perumal et al., 2018; Tigani et al., 2005; Vicenti et al., 2019) have been inconclusive at best and, therefore, it has neither greatly influenced nor improved clinical practice. Likewise, a recently published systematic review and metanalysis on the effects of dynamisation on fracture healing by

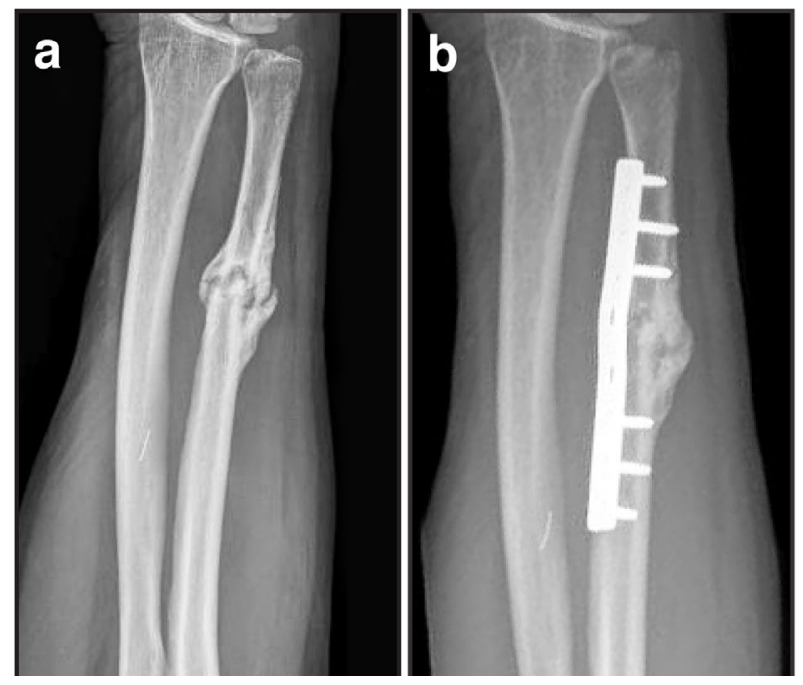

Fig. 2. Radiographic images of distal ulna diaphyseal fracture. (a) A hypertrophic non-union formed when managed non-operatively as a result of too much micromotion at the fracture site. (b) The fracture healed spontaneously without bone grafting after open reduction internal fixation, and compression plating achieved rigid fixation. 
Ferreira et al. (2020) concluded that the diversity in dynamisation strategies, the variation in the timing of dynamisation and the ambivalence of the results precludes any definitive conclusions. Therefore, further research is necessary to improve fracture healing when using dynamisation before specific recommendations can be made.

Over the past decade, the revolutionary concept of reverse dynamisation (Fig. 3), a modern and refined perspective on Stephan Perren's strain theory, has
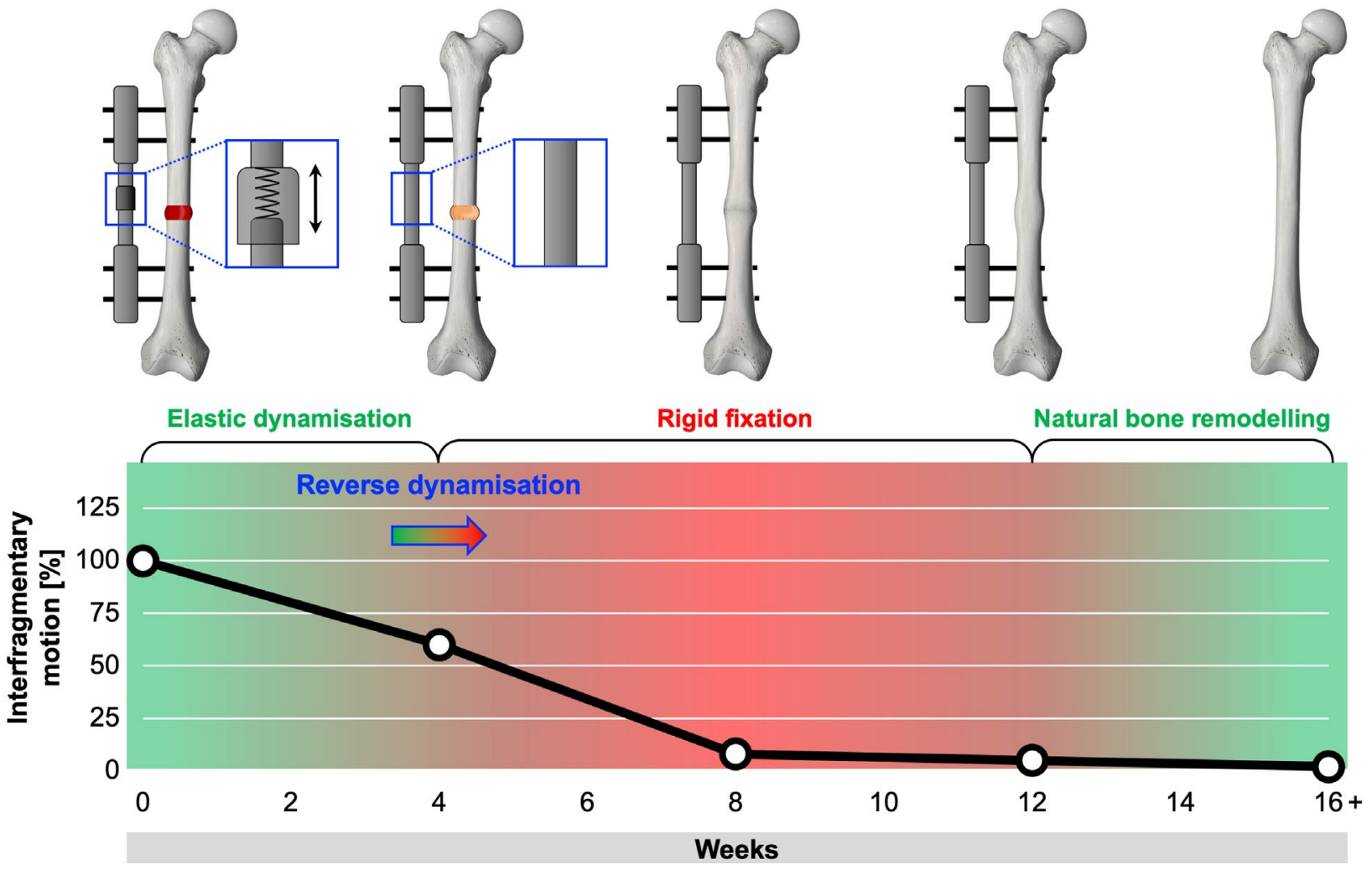

Fig. 3. Schema illustrateing the reverse dynamisation regimen, which is a modernised perspective on Stephan Perren's strain theory on how to achieve more rapid and reliable bone healing. The hypothesis is that a fracture initially stabilised using flexible fixation (elastic dynamisation; indicated in green) allows for micromotion and encourages abundant cartilaginous callus formation. Once substantial callus has formed, which is anywhere from 2 to 4 weeks, the stabilisation is converted to a rigid configuration (red) to prevent the disruption of neovascularisation, which then allows bone to rapidly remodel at a faster rate. After complete bone healing occurs, the fixator is removed (at around 12 weeks) to allow for natural bone remodelling to restore its anatomical shape and physical properties.

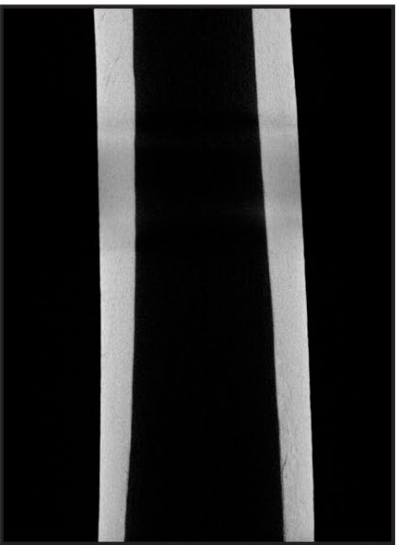

IT

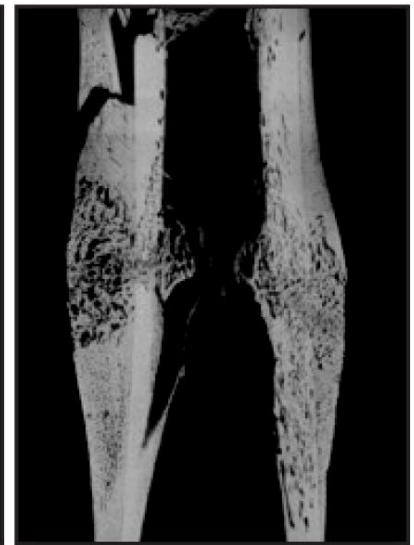

SF

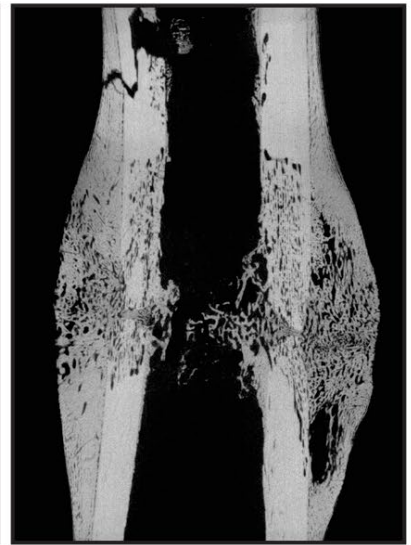

DF

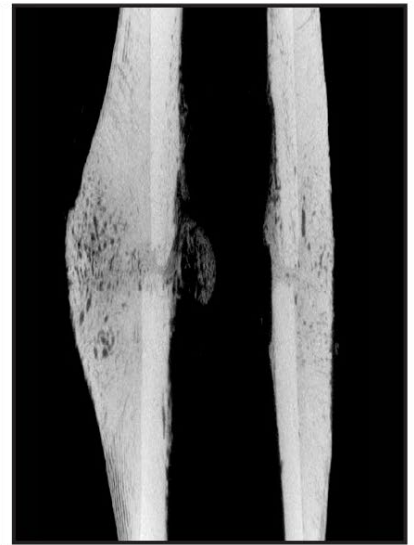

RDF

Fig. 4. Two-dimensional (2D) coronal micro-computed tomography images of intact tibia (IT) and $2 \mathrm{~mm}$ tibial osteotomies in a goat after 8 weeks of healing. Osteotomies were stabilised with static fixation (SF), dynamic fixation (DF) and reverse dynamisation (RD; dynamic fixation for 3 weeks and at 3 weeks the fixators were converted to static/rigid fixation) protocols (Glatt et al., 2020). Scale bar $=5 \mathrm{~mm}$. 
been slowly gathering momentum (Bartnikowski et al., 2017; Glatt et al., 2016a; Glatt et al., 2016b; Glatt et al., 2012b; Glatt et al., 2020; Glatt et al., 2016c; Tetsworth and Glatt, 2019). With this strategy, the mechanical environment of the fracture site is actively manipulated during the early stage of healing in an attempt to optimise and accelerate the progression of bone towards union. It is based on the hypothesis that a fracture initially stabilised using flexible fixation allows for micromotion and encourages abundant cartilaginous callus formation. Once substantial callus has formed, the stabilisation is converted to a rigid configuration to prevent the disruption of neovascularisation, which then allows the bone to rapidly remodel at a faster rate and to restore its anatomical shape and physical properties. A growing body of experimental research supports reverse dynamisation and demonstrates the potential to optimise rapid bone healing (Bartnikowski et al., 2017; Glatt et al., 2016a; Glatt et al., 2016b; Glatt et al., 2012b; Glatt et al., 2020; Glatt et al., 2016c). For example, studies in rat models have demonstrated that early modulation (7-14 d) of the mechanical environment, by first stabilising the defect under conditions of low stiffness and then imposing high stiffness after provisional callus formation is observed, significantly accelerates bone healing (Bartnikowski et al., 2017). By contrast, a study by Claes et al. (2009) in the same animal model found that dynamisation at $7 \mathrm{~d}$, when initially high stiffness fixation is followed by low stiffness fixation, is very detrimental to bone healing. Interestingly, using either the conventional dynamisation or reverse dynamisation regimen during the late phase of bone healing (remodelling) had very similar results (Bartnikowski et al., 2017; Claes et al., 2011). These outcomes are not surprising
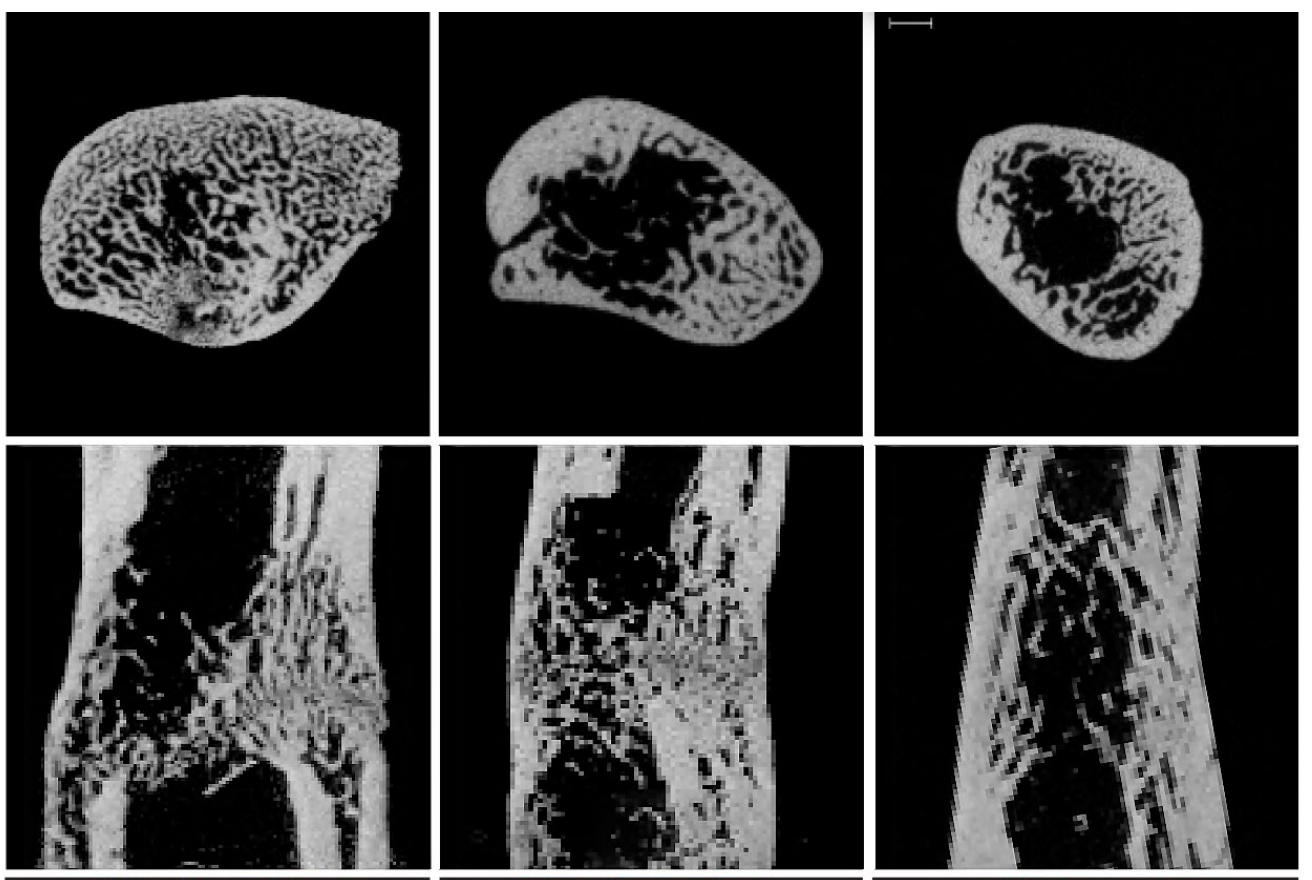

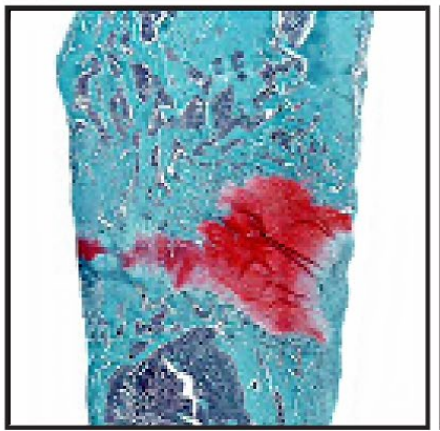

Flexible fixation

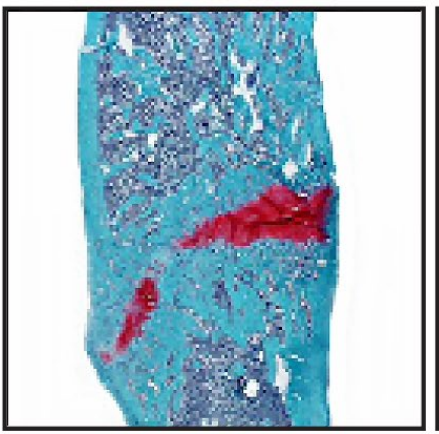

Rigid fixation
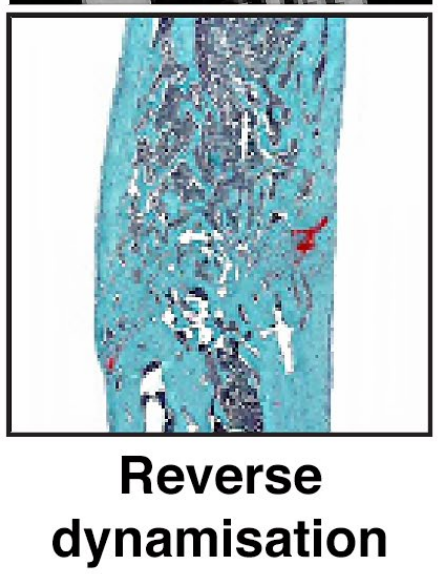

Fig. 5. Micro-computed tomography and histology images illustrating the healing of $5 \mathrm{~mm}$ segmental defects in the rat femur treated with $5.5 \mu$ of BMP-2, with flexible external fixation, rigid external fixation or reverse dynamisation after 8 weeks of treatment (Glatt et al., 2016a). Micro-computed tomography images of cross-sectional central part of the defect (top row) and coronal plane of the defect (middle row). Histological sections were stained with safranin orange-fast green (bottom row, scale bar $=1 \mathrm{~mm}$ ). Flexible fixation = low stiffness fixator $(114 \mathrm{~N} / \mathrm{mm})$; rigid fixation = high stiffness fixator $(254 \mathrm{~N} / \mathrm{mm})$; reverse dynamisation $=$ flexible to rigid stiffness fixation at 2 weeks (Glatt et al., 2012a). 
since two ex vivo experimental studies (Gardner et al., 1996; Glatt et al., 2012a) have independently concluded that when stiff intra-fracture materials are used to simulate the remodelling stage of healing, the stiffness of the fixator contributes very little to the overall stability of the construct. Instead, most of the axial loading is transferred mainly through the stiffened intra-fracture material, with the fixator only minimally contributing to the stability. Furthermore, a recent study in a goat $2 \mathrm{~mm}$ osteotomy model also confirmed that the reverse dynamisation regimen accelerates healing and remodelling of tibiae compared to the static/rigid and the dynamic/ flexible fixation groups (Fig. 4) (Glatt et al., 2020). This was achieved by initially providing flexible fixation for 3 weeks to allow early micromotion, thereby encouraging the maximal amount of soft callus formation. Once abundant callus formation was visible radiographically, the mechanical environment was converted to a more rigid configuration, in contradistinction to conventional dynamisation that has been utilised for the past several decades.

The results from these reverse dynamisation studies easily explain the inconsistent reports in the literature when using the conventional dynamisation regimen. It appears mainly to be related to the type of dynamisation regimen used and the timing of its introduction. For instance, axial dynamisation allows for a degree of collapse or compression of the fracture fragments and, when used on 1-3 mm osteotomies during early stages of healing, an axially dynamised construct essentially becomes more rigid, as the bone surfaces make contact. These conditions, in fact, inadvertently approximate the reverse dynamisation regimen and these studies have, therefore, observed beneficial effects on bone healing (Barquet et al., 1992; Egger et al., 1993; Foxworthy and Pringle, 1995; Larsson et al., 2001). Elastic dynamisation allows for temporary deformation at the fracture site under physiological load and recovery of the original fracture dimensions during unloading. Therefore, when elastic dynamisation is used during the initial stages of healing, the construct is flexible, thus providing a certain amount of micromotion at the fracture site, initiating callus formation (Bartnikowski et al., 2017; Glatt et al., 2016a; Glatt et al., 2012b). Again, it is not surprising that elastic dynamisation applied during the reparative stage of healing instead resulted in fracture non-unions (Claes et al., 2009; Foxworthy and Pringle, 1995). Similarly, Kenwright and Gardner (1998) have also previously demonstrated that excessive motion after a callus has formed may be
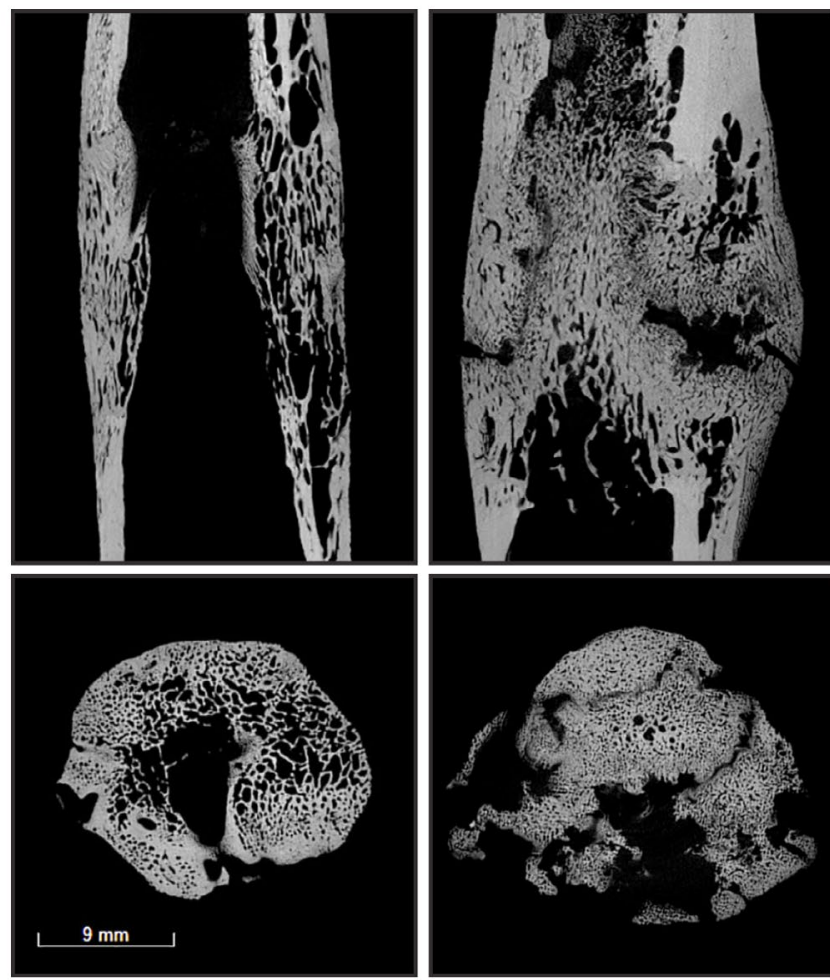

SF

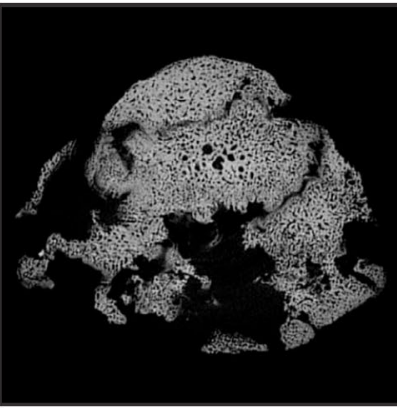

DF
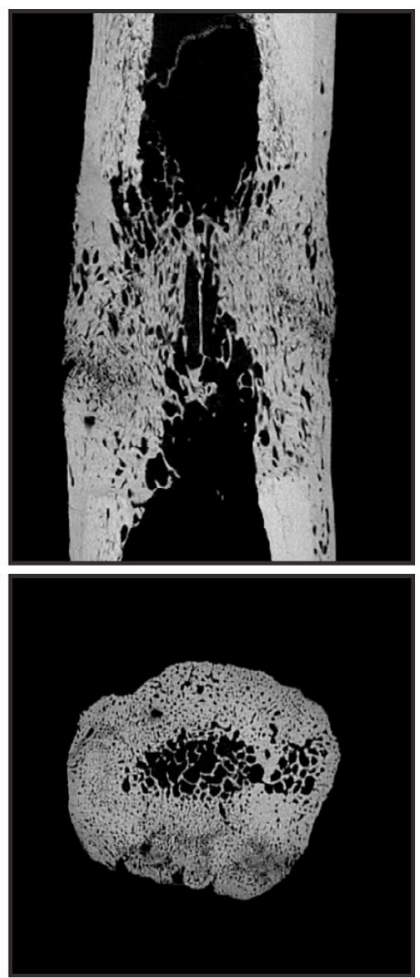

RDF

Fig. 6. Micro-computed tomography images of a goat $20 \mathrm{~mm}$ distraction osteogenesis model stabilised with static fixation (SF; constant rigid fixation group), dynamic fixation (DF; flexible fixation group, allowing $2 \mathrm{~mm}$ elastic/axial compression during the study period) and reverse dynamisation (RDF; started with flexible fixation, allowing $2 \mathrm{~mm}$ elastic/axial compression and at 6 weeks was converted to static fixation for the remaining study period) protocols. The distraction commenced on the $6^{\text {th }}$ post-operative day and continued until $20 \mathrm{~mm}$ distraction was achieved, which was 6 weeks after surgery. At 6 weeks, the RD group was converted from dynamic fixation to static fixation. All of the groups were allowed an 8-week consolidation period. 2D sagittal (top row) and cross-sectional images illustrating the central area of the regenerate bone (bottom row). Scale bar $=9 \mathrm{~mm}$. 
detrimental and can disrupt the nascent fracture gap tissue and delay the remodelling process.

The reverse dynamisation regimen has demonstrated similar results in large segmental defects and distraction osteogenesis/limb lengthening of bone. For example, the effects of fixator stiffness on the healing of large bone defects treated with BMP-2 was investigated in a rat large segmental defect model (Fig. 5). The hypothesis was that manipulation of the mechanical environment as healing progresses could enhance the healing of large osseous defects; specifically, that accelerated healing would be achieved by first stabilising the defect under conditions of low stiffness and, then, converting to high stiffness conditions once callus formation was evident radiographically. This study convincingly confirmed the healing of large bone defects is in fact very highly responsive to the ambient mechanical environment and that by altering fixation stability the rate and quality of healing can be predictably manipulated (Glatt et al., 2012c). Based on these observations, a subsequent study determined whether the dose of BMP-2 could be reduced without compromising the healing process when using this enhanced mechanical environment (Glattet al., 2016a). Although the initial healing was slightly delayed, forming a smaller callus throughout the healing period, the study demonstrated the quality of healing bone was similar, or slightly superior, to that treated with the higher dose of BMP-2. Of further interest, when the dose of BMP-2 was insufficient, the same study revealed that healing did not occur regardless of which stiffness fixation device was used. Although preliminary, similar results are being observed in an ongoing study using a goat $2 \mathrm{~cm}$ distraction osteogenesis model (Fig. 6). The basis of this study is that providing a small amount of micromotion (elastic dynamisation) during the bone lengthening period would promote larger and more rapid callus formation of the regenerate. Once the distraction to the desired length has been completed, the fixator frame is altered to a more rigid fixation during the consolidation period to accelerate remodelling of the regenerated bone. The initial findings of this study are striking and suggest that the proposed regimen of reverse dynamisation significantly accelerates regenerate remodelling (unpublished data).

Unfortunately, high quality clinical data regarding the role of reverse dynamisation and fracture healing remains scarce and most reports are unpublished or anecdotal. Although other trials are underway, there has been only one published study that has investigated the reverse dynamisation regimen in humans. Examining adult tibial fractures stabilised with external fixation, Howard et al. (2013) demonstrated that a standard protocol, allowing initial axial micromovement from 2 to 4 weeks after surgery followed by more rigid stabilisation, indeed accelerated fracture healing. When treating isolated closed or grade I open tibial fractures, faster healing was associated with a reduction in the average time to removal of the fixator in the dynamisation group ( 11 weeks), compared to the standard of care ( $\sim 22$ weeks). In an effort to better define the role and the optimal clinical parameters for reverse dynamisation, Dr Glatt is currently actively coordinating a series of clinical research projects at various sites around the globe.

\section{Conclusions}

New studies are rapidly expanding our knowledge about the influence of modulating the mechanical environment on bone healing. Although instability is often clinically associated with non-unions, increasing evidence suggests that providing a small amount of flexibility or micromotion at the fracture gap during the initial stage of healing has beneficial effects on bone healing and is challenging the clinical consensus that fracture stability/rigidity is critical. Furthermore, what has also become evident is that micromotion or flexible fixation at the fracture site is only required during the initial phase of the healing process, anywhere from 2 to 4 weeks, before converting to more rigid fixation. Importantly, this was demonstrated not only in the studies of small animal models (Bartnikowski et al., 2017; Glatt et al., 2016a; Glatt et al., 2012b) but has also been observed in a large animal model (Glatt et al., 2020) as well as in one supportive clinical study (Howard et al., 2013).

These findings support the concept that bone healing inevitably reflects a very complex relationship previously characterised as the concert between biology and biomechanics, and when these two elements are in harmony bone healing most often proceeds melodically to a satisfying union (Glatt et al., 2016b). In the diamond concept of fracture healing, the mechanical environment is considered an equal contributor, together with osteogenic cells, growth factors and osteoconductive scaffolds (Giannoudis et al., 2007). However, in the authors' opinion, the biomechanics should be considered the most important factor because the mechanical environment almost certainly orchestrates the nature of the biological response (Glatt et al., 2016b). Consequently, the biological response can never overcome a mechanical environment that does not support adequate bone growth, even when augmented with exogenous factors. Therefore, whenever the biology and biomechanics act in symphony, bone is able to heal rapidly and predictably, as has already been demonstrated by several studies using the reverse dynamisation regimen.

\section{Future perspectives}

As Perren recognised, biomechanical stability probably plays the most critical role in fracture healing and surgical decisions such as implant choice should be guided by an understanding 
of this fundamental principle. His strain theory qualitatively describes the bone healing response with respect to the mechanical environment and the strain tolerance of the various tissue types involved is the prime determinant. However, the optimal amount of strain required for different healing phases is still unknown and further studies defining these parameters more explicitly remain vitally important. More recent studies have also confirmed that successful regeneration and healing of bone relies on a synergy between the various biological factors and mechanical forces, governed by the timing and spatial relationship of their introduction. The reverse dynamisation regimen provides a modern perspective on Stephan Perren's strain theory and perhaps holds the key to tipping the balance in favour of a more rapid and reliable union in settings including acute fractures, osteotomies, non-unions and other circumstances where it is necessary to regenerate bone. Evolving technologies may soon be able to measure the progression of callus formation and stiffness as the healing progresses, and these should enhance our ability to optimally modulate the biomechanics and willfully influence the biology. Promising technologies currently in development include electromagnetic antennas, implantable microelectromechanical sensors and smart internal plates containing strain sensors able to measure tissue stiffness within the fracture gap in real-time. This will require further development of plates and nails that provide a mechanism to carefully manipulate their mechanical properties without the need for further surgery, perhaps employing nitinol alloy to actively change the local mechanical environment in favour of a more rapid and robust fracture healing. While future challenges still remain in understanding and optimising the nature of these mechanical cues and the biological responses to them at various stages of bone healing, the initial results from ongoing investigations have been very encouraging and it will be interesting to observe what these continuing studies will reveal.

\section{Acknowledgments}

We would like to thank Dr Anna Woloszyk (The University of Texas Health Science Centre, San Antonio, TX, USA) for making Fig. 3. Furthermore, we would also like to thank Miva Stock Photography (Boston, MA, USA) for creating illustrations for Fig. 1 and 3. Dr Evans's research is supported in part by the John and Posy Krehbiel Professorship in Orthopedics.

The authors declare that they have no competing interests.

\section{References}

Akeson WH, Woo SL, Rutherford L, Coutts RD, Gonsalves M, Amiel D (1976) The effects of rigidity of internal fixation plates on long bone remodeling. A biomechanical and quantitative histological study. Acta Orthop Scand 47: 241-249.

Aro HT, Chao EY (1993) Bone-healing patterns affected by loading, fracture fragment stability, fracture type, and fracture site compression. Clin Orthop Relat Res: 8-17.

Aro HT, Kelly PJ, Lewallen DG, Chao EY (1990) The effects of physiologic dynamic compression on bone healing under external fixation. Clin Orthop Relat Res: 260-273.

Augat P, Burger J, Schorlemmer S, Henke T, Peraus M, Claes L (2003) Shear movement at the fracture site delays healing in a diaphyseal fracture model. J Orthop Res 21: 1011-1017.

Augat P, Margevicius K, Simon J, Wolf S, Suger G, Claes L (1998) Local tissue properties in bone healing: influence of size and stability of the osteotomy gap. J Orthop Res 16: 475-481.

Augat P, Merk J, Ignatius A, Margevicius K, Bauer G, Rosenbaum D, Claes L (1996) Early, full weightbearing with flexible fixation delays fracture healing. Clin Orthop Relat Res: 194-202.

Augat P, Simon U, Liedert A, Claes L (2005) Mechanics and mechano-biology of fracture healing in normal and osteoporotic bone. Osteoporos Int 16 Suppl 2: S36-43.

Barquet A, Massaferro J, Dubra A, Milans C, Castiglioni O (1992) The dynamic ASIF-BM tubular external fixator in the treatment of open fractures of the shaft of the tibia. Injury 23: 461-466.

Bartnikowski N, Claes LE, Koval L, Glatt V, Bindl R, Steck R, Ignatius A, Schuetz MA, Epari DR (2017) Modulation of fixation stiffness from flexible to stiff in a rat model of bone healing. Acta Orthop 88: 217222.

Basumallick MN, Bandopadhyay A (2002) Effect of dynamization in open interlocking nailing of femoral fractures. A prospective randomized comparative study of 50 cases with a 2-year follow-up. Acta Orthop Belg 68: 42-48.

Bishop NE, van Rhijn M, Tami I, Corveleijn R, Schneider E, Ito K (2006) Shear does not necessarily inhibit bone healing. Clin Orthop Relat Res 443: 307314.

Bottlang M, Doornink J, Lujan TJ, Fitzpatrick DC, Marsh JL, Augat P, von Rechenberg B, Lesser M, Madey SM (2010) Effects of construct stiffness on healing of fractures stabilized with locking plates. J Bone Joint Surg Am 92 Suppl 2: 12-22.

Carter DR, Beaupre GS, Giori NJ, Helms JA (1998) Mechanobiology of skeletal regeneration. Clin Orthop Relat Res: S41-55. DOI: 10.1097/00003086199810001-00006.

Carter DR, Blenman PR, Beaupre GS (1988) Correlations between mechanical stress history and tissue differentiation in initial fracture healing. J Orthop Res 6: 736-748.

Carter DR, Wong M (1988) The role of mechanical loading histories in the development of diarthrodial joints. J Orthop Res 6: 804-816. 
Chao EY, Aro HT, Lewallen DG, Kelly PJ (1989) The effect of rigidity on fracture healing in external fixation. Clin Orthop Relat Res: 24-35.

Cheal EJ, Mansmann KA, DiGioia AM, 3rd, Hayes WC, Perren SM (1991) Role of interfragmentary strain in fracture healing: ovine model of a healing osteotomy. J Orthop Res 9: 131-142.

Claes L, Augat P, Suger G, Wilke HJ (1997) Influence of size and stability of the osteotomy gap on the success of fracture healing. J Orthop Res 15: 577-584.

Claes L, Blakytny R, Besse J, Bausewein C, Ignatius A, Willie B (2011) Late dynamization by reduced fixation stiffness enhances fracture healing in a rat femoral osteotomy model. J Orthop Trauma 25: 169174.

Claes L, Blakytny R, Gockelmann M, Schoen M, Ignatius A, Willie B (2009) Early dynamization by reduced fixation stiffness does not improve fracture healing in a rat femoral osteotomy model. J Orthop Res 27: 22-27.

Claes LE, Heigele CA (1999) Magnitudes of local stress and strain along bony surfaces predict the course and type of fracture healing. J Biomech 32: 255-266.

Claes LE, Heigele CA, Neidlinger-Wilke C, Kaspar D, Seidl W, Margevicius KJ, Augat P (1998) Effects of mechanical factors on the fracture healing process. Clin Orthop Relat Res: S132-147. DOI: 10.1097/00003086-199810001-00015.

De La Vega RE, De Padilla CL, Trujillo M, Quirk N, Porter RM, Evans CH, Ferreira E (2018) Contribution of implanted, genetically modified muscle progenitor cells expressing BMP-2 to new bone formation in a rat osseous defect. Mol Ther 26: 208-218.

Domb BG, Sponseller PD, Ain M, Miller NH (2002) Comparison of dynamic versus static external fixation for pediatric femur fractures. J Pediatr Orthop 22: 428-430.

Egger EL, Gottsauner-Wolf F, Palmer J, Aro HT, Chao EY (1993) Effects of axial dynamization on bone healing. J Trauma 34: 185-192.

Epari DR, Schell H, Bail HJ, Duda GN (2006) Instability prolongs the chondral phase during bone healing in sheep. Bone 38: 864-870.

Ferreira N, Tanwar YS, Burger M (2020) The effect of fixation dynamization on fracture healing: a systematic review. J Limb Lengthen Reconstr 6: 7-12.

Foxworthy M, Pringle RM (1995) Dynamization timing and its effect on bone healing when using the Orthofix Dynamic Axial Fixator. Injury 26: 117-119.

Gardner TN, Evans M, Kenwright J (1996) The influence of external fixators on fracture motion during simulated walking. Med Eng Phys 18: 305-313.

Giannoudis PV, Einhorn TA, Marsh D (2007) Fracture healing: the diamond concept. Injury 38 Suppl 4: S3-6.

Glatt V, Bartnikowski N, Quirk N, Schuetz M, Evans C (2016a) Reverse dynamization: Influence of fixator stiffness on the mode and efficiency of large- bone-defect healing at different doses of rhBMP-2. J Bone Joint Surg Am 98: 677-687.

Glatt V, Evans CH, Matthys R (2012a) Design, characterisation and in vivo testing of a new, adjustable stiffness, external fixator for the rat femur. Eur Cell Mater 23: 289-299.

Glatt V, Evans CH, Tetsworth K (2016b) A concert between biology and biomechanics: the influence of the mechanical environment on bone healing. Front Physiol 7: 678. DOI: 10.3389/fphys.2016.00678.

Glatt V, Miller M, Ivkovic A, Liu F, Parry N, Griffin D, Vrahas M, Evans C (2012b) Improved healing of large segmental defects in the rat femur by reverse dynamization in the presence of bone morphogenetic protein-2. J Bone Joint Surg Am 94: 2063-2073.

Glatt V, Miller M, Ivkovic A, Liu F, Parry N, Griffin D, Vrahas M, Evans C (2012c) Improved healing of large segmental defects in the rat femur by reverse dynamization in the presence of bone morphogenetic protein-2. J Bone Joint Surg Am 94: 2063-2073.

Glatt V, Samchukov M, Cherkashin A, Iobst C (2020) Reverse dynamization accelerates bonehealing in a large-animal osteotomy model. J Bone Joint Surg Am 103: 257-263.

Glatt V, Tepic S, Evans C (2016c) Reverse dynamization: a novel approach to bone healing. J Am Acad Orthop Surg 24: e60-61.

Goodship AE, Cunningham JL, Kenwright J (1998) Strain rate and timing of stimulation in mechanical modulation of fracture healing. Clin Orthop Relat Res: S105-115. DOI: 10.1097/00003086-19981000100012.

Goodship AE, Kenwright J (1985) The influence of induced micromovement upon the healing of experimental tibial fractures. J Bone Joint Surg Br 67: 650-655.

Goodship AE, Lawes TJ, Rubin CT (2009) Lowmagnitude high-frequency mechanical signals accelerate and augment endochondral bone repair: preliminary evidence of efficacy. J Orthop Res 27: 922-930.

Hagiwara $Y$, Dyment NA, Jiang $X$, Jiang Ping H, Ackert-Bicknell C, Adams DJ, Rowe DW (2015) Fixation stability dictates the differentiation pathway of periosteal progenitor cells in fracture repair. J Orthop Res 33: 948-956.

Howard CB, Leibergal M, Elishoov O, Matan Y, Segal D, Porat S (2013) Can changing the mechanical environment increase the speed of fracture healing? A pilot study in tibial fractures. J Trauma Treat 2: 1-5.

Hu DP, Ferro F, Yang F, Taylor AJ, Chang W, Miclau T, Marcucio RS, Bahney CS (2017) Cartilage to bone transformation during fracture healing is coordinated by the invading vasculature and induction of the core pluripotency genes. Development 144: 221-234.

Huang KC, Tong KM, Lin YM, Loh el W, Hsu CE (2012) Evaluation of methods and timing in nail dynamisation for treating delayed healing femoral shaft fractures. Injury 43: 1747-1752.

Ignatius A, Blessing $\mathrm{H}$, Liedert A, Schmidt $\mathrm{C}$, Neidlinger-Wilke C, Kaspar D, Friemert B, Claes 
L (2005) Tissue engineering of bone: effects of mechanical strain on osteoblastic cells in type I collagen matrices. Biomaterials 26: 311-318.

Kenwright J, Gardner T (1998) Mechanical influences on tibial fracture healing. Clin Orthop Relat Res: S179-190. DOI: 10.1097/00003086-19981000100019.

Larsson S, Kim W, Caja VL, Egger EL, Inoue N, Chao EY (2001) Effect of early axial dynamization on tibial bone healing: a study in dogs. Clin Orthop Relat Res: 240-251. DOI: 10.1097/00003086-20010700000033.

Litrenta J, Tornetta P 3rd, Vallier H, Firoozabadi R, Leighton R, Egol K, Kruppa C, Jones CB, Collinge C, Bhandari M, Schemitsch E, Sanders D, Mullis B (2015) Dynamizations and exchanges: Success rates and indications. J Orthop Trauma 29: 569-573.

Matsushita T, Kurokawa T (1998) Comparison of cyclic compression, cyclic distraction and rigid fixation. Bone healing in rabbits. Acta Orthop Scand 69: 95-98.

Papakostidis C, Psyllakis I, Vardakas D, Grestas A, Giannoudis PV (2011) Femoral-shaft fractures and nonunions treated with intramedullary nails: the role of dynamisation. Injury 42: 1353-1361.

Pauwels F (1960) [A new theory on the influence of mechanical stimuli on the differentiation of supporting tissue. The tenth contribution to the functional anatomy and causal morphology of the supporting structure.]. Z Anat Entwicklungsgesch 121: $478-515$.

Perren SM (1979) Physical and biological aspects of fracture healing with special reference to internal fixation. Clin Orthop Relat Res: 175-196.

Perren SM (2002) Evolution of the internal fixation of long bone fractures. The scientific basis of biological internal fixation: choosing a new balance between stability and biology. J Bone Joint Surg Br 84: 1093-1110.

Perren SM (2008) Fracture healing. The evolution of our understanding. Acta Chir Orthop Traumatol Cech 75: 241-246.

Perren SM, Cordey J (1980) The concept of interfragmentary strain. current concepts of internal fixation of fractures. Springer-Verlag, Berlin. pp: 6377.

Perren SM, Fernandez A, Regazzoni P (2015) Understanding fracture healing biomechanics ased on the "strain" concept and its clinical applications. Acta Chir Orthop Traumatol Cech 82: 253-260.

Perren SM, Rahn BA (1980) Biomechanics of fracture healing. Can J Surg 23: 228-232.

Perumal R, Shankar V, Basha R, Jayaramaraju D, Rajasekaran S (2018) Is nail dynamization beneficial after twelve weeks - an analysis of 37 cases. J Clin Orthop Trauma 9: 322-326.

Probst A, Jansen H, Ladas A, Spiegel HU (1999) Callus formation and fixation rigidity: a fracture model in rats. J Orthop Res 17: 256-260.

Roux W (1881a) Der Kampf der Theile im Organismus. Ein Beitrag zur vervollständigung der mechanischen Zweckmässigkeitslehre. Leipzig. DOI: 10.5962/bhl.title.1491.

Roux W (1881b) Der Zuchtende Kampf der Teile, oder die "Teilauslese" im Organismus. In: Abhandlungen über Entwickelungsmechanik der Organismus. Editor: Krosigk E. Wilhelm Engelmann, Leipzig. pp: 135-422.

Tetsworth K, Glatt V (2019) Tipping the balance: manipulating the mechanical environment by reverse dynamization can accelerate bone healing. J Limb Lengthen Reconstr 5: 1-3.

Tigani D, Fravisini M, Stagni C, Pascarella R, Boriani S (2005) Interlocking nail for femoral shaft fractures: is dynamization always necessary? Int Orthop 29: 101-104.

Uhthoff HK, Dubuc FL (1971) Bone structure changes in the dog under rigid internal fixation. Clin Orthop Relat Res 81: 165-170.

Utvag SE, Korsnes L, Rindal DB, Reikeras O (2001) Influence of flexible nailing in the later phase of fracture healing: strength and mineralization in rat femora. J Orthop Sci 6: 576-584.

Utvag SE, Reikeras O (1998) Effects of nail rigidity on fracture healing. Strength and mineralisation in rat femoral bone. Arch Orthop Trauma Surg 118: 7-13.

Utvag SE, Rindal DB, Reikeras O (1999) Effects of torsional rigidity on fracture healing: strength and mineralization in rat femora. J Orthop Trauma 13: 212-219.

Vicenti G, Bizzoca D, Carrozzo M, Nappi V, Rifino F, Solarino G, Moretti B (2019) The ideal timing for nail dynamization in femoral shaft delayed union and non-union. Int Orthop 43: 217-222.

Yang L, Tsang KY, Tang HC, Chan D, Cheah KS (2014) Hypertrophic chondrocytes can become osteoblasts and osteocytes in endochondral bone formation. Proc Natl Acad Sci U S A 111: 12097-12102.

\section{Discussion with Reviewers}

Reviewer: Is Stephan Perren's strain theory (SPST) applicable to all types of fractures, more precise diaphyseal and metaphyseal fractures? How is it affected by the inter fragmentary gap size?

Authors: SPST was originally proposed with respect to diaphyseal fracture healing. Unfortunately, there is a gap in our knowledge and differences in fracture healing responses between either metaphyseal or diaphyseal bone have not yet been explored in detail. Similarly, the mechanical conditions also differ dramatically between diaphyseal and metaphyseal locations and have not yet been examined. However, we would speculate that SPST is also applicable to metaphyseal fractures, because theoretically as long as a certain amount of micromotion is provided, not too much and not too little, metaphyseal fractures might heal even more rapidly. As always, this depends upon a delicate balance between the gap size and the fracture fixation stiffness. 
The size of the fracture gap is of critical importance with respect to both the biology and biomechanic, although it was not defined explicitly in SPST. Consider first an anatomically reduced fracture with a very small gap rigidly fixed with a wellpositioned plate, where the strain is low and therefore conducive to primary bone healing. If one were to take the same fracture stabilised in the identical fashion but with some distraction, the strain would be potentially reduced, suggesting that primary healing will continue. However, a larger distraction often leads to instability and greater IFM, resulting in secondary bone formation. This is consistent with what is observed clinically, where distraction and malreduction result in instability, and the strain conditions are then more conducive to secondary fracture healing. Finally, consider the situation where the fracture is grossly malreduced but rigidly fixed, and there may be a gap of $10 \mathrm{~mm}$ or more. Although the low strain favours primary healing, the gap is now far too large to bridge, leading to a fibrous non-union. This is ultimately the result of a complete discord between the mechanical conditions and the ensuing biological response. Then again, in metaphyseal bone stabilised as a bridging construct, this gap could still potentially heal spontaneously in a healthy patient with a good soft-tissue envelope and intact periosteum.

In contrast, reverse dynamisation encompasses both the biology and the biomechanics, this is conceptually more attractive than SPST alone. By providing a flexible initial stabilisation, the greater strain encourages secondary bone healing and abundant callus formation. Monitoring the biological response radiographically provides a feedback to determine the point when conversion to more rigid fixation is indicated, and this can be optimised. This remains true in all situations, regardless of the gap size, the location in the bone (diaphyseal or metaphyseal) or even whether the fixation is through a plate, an intramedullary nail or an external fixator. Of course, the biological response is still limited in its ability to bridge larger gaps, but RD allows the clinician to prospectively determine when, and in what matter, to intervene with the goal of rapid, consistent bone healing.

Farshid Guilak: Can the authors speculate on how this method could be advanced to the next stage to optimise the tissue responses? For example, would it be possible to have real-time measurements of repair tissue mechanics/quality that then informs the decision to change the level of dynamisation? How would this method be used in combination with biological approaches (e.g. gene therapy, stem cells, growth factor delivery) to enhance repair?

Authors: The only way to determine the quality of the tissue within the fracture site currently is by X-ray, which has been shown to be quite reliable but of course not optimal. However, studies are under way to determine the optimal amount of micromotion that is required during the early stages of healing to induce rapid and robust callus formation, while at the same time avoiding excessive micromotion within the fracture gap, which has the potential to generate a delayed union or non-union. Furthermore, new technologies are in development that will be able to measure the progression of callus formation and stiffness as the healing progresses. This includes technologies employing electromagnetic antennas, implantable microelectromechanical sensors and smart internal plates that have strain sensors able to measure tissue stiffness within the fracture gap in real-time and provide this information via Bluetooth to a mobile app. Then, this will let the treating surgeon know when it is time to change the fixator stiffness to more rigid fixation. Likewise, smart implants using internal plates and nails that are made out of a memory metal such nitinol, which remembers its high temperature shape from when heated, can be adjusted by slight changes in alloy composition and through heat treatment.

When it comes to using biologics there should not be any difference concerning the effects of reverse dynamisation and how it would influence the healing outcomes. The only difference will be in the amount of micromotion that would be applied during the initial stage of healing (percentage of strain will be the same, less than $10 \%$ ), which has nothing to do with the biologics but rather the gap size. The callus formation would still follow a similar pattern as normally healing fractures, because the healing progression, the rate of healing and the phases of the healing process are very similar with and without the use of biologics. However, there is the possibility that certain biologics would accelerate or amplify the regenerative effects of reverse dynamisation, and vice versa. For critical sized defects, a biological stimulus, such as BMP-2, is necessary to initiate healing; reverse dynamisation alone cannot do this.

Editor's note: The Guest Editor responsible for this paper was Anita Ignatius. 